\title{
Non-Linear Energy Harvesting in RIS-assisted URLLC Networks for Industry Automation
}

This paper was downloaded from TechRxiv (https://www.techrxiv.org).

\section{LICENSE}

CC BY 4.0

SUBMISSION DATE / POSTED DATE

26-07-2021 / 30-07-2021

\section{CITATION}

Dhok, Shivani; Raut, Prasanna; Sharma, Prabhat Kumar; Singh, Keshav; Li, Chih-Peng (2021): Non-Linear Energy Harvesting in RIS-assisted URLLC Networks for Industry Automation. TechRxiv. Preprint. https://doi.org/10.36227/techrxiv.15057600.v1

$\mathrm{DOI}$

10.36227/techrxiv.15057600.v1 


\title{
Non-Linear Energy Harvesting in RIS-assisted URLLC Networks for Industry Automation
}

\author{
Shivani Dhok, Student Member, IEEE, Prasanna Raut, Member, IEEE, Prabhat Kumar Sharma, Senior \\ Member, IEEE, Keshav Singh, Member, IEEE, and Chih-Peng Li, Fellow, IEEE
}

\begin{abstract}
A reconfigurable intelligent surface (RIS)-assisted wireless communication system with non-linear energy harvesting (EH) and ultra-reliable low-latency constraints is considered for its possible applications in industrial automation. A distant datacenter (DC) communicates with the multiple destination machines with the help of a full-duplex (FD) server machine (SM) and RIS. Assuming the deficiency of enough transmission power at the FDSM, the SM is considered in the near vicinity of the destinations in the industry to forward the data received from the distant DC. The reception at SM is assisted by the RIS and a non-linear hybrid power-time splitting (PTS) based EH receiver architecture is adopted to extend the lifespan of SM, thus increasing network lifetime. The scheduling of multiple destinations is done by SM based on the considered selection criteria namely, random (RND) scheduling, absolute (ABS) channel-power-based (CPB) scheduling and normalized (NRM) CPB scheduling. The end-toend performance of the considered FD RIS-assisted network is analyzed, and the expressions for the block error rate (BLER) for all scheduling schemes are derived. Moreover, the effects of number of RIS elements, packet size, channel uses on the system performance are analyzed for the considered ultra-reliable and low-latency communication (URLLC) network. The scheduling fairness of all the scheduling schemes is also analyzed to study the performance-fairness trade-off. The derived analytical results are verified through Monte-Carlo simulations.
\end{abstract}

Index Terms-Block error rate, non-linear energy harvesting, full-duplex, industry automation, reconfigurable intelligent surfaceS, scheduling, ultra-reliable low-latency communication.

\section{INTRODUCTION}

$\mathbf{R}$ ECENT evolution in the wireless technologies has led to a significant demand for increased data-rates, connectivity and spectral efficiency. It is forecast that the number of connected devices would reach to 36048 million by 2025 ,

S. Dhok and P. K. Sharma are with the Department of Electronics \& Communication Engineering at Visvesvaraya National Institute of Technology, Nagpur, India. Part of work was carried out by S. Dhok during her internship at National Sun Yat-sen University, Taiwan. (e-mail: shivanid17499@gmail.com, prabhatsharma@ece.vnit.ac.in ).

P. Raut was with the Institute of Communications Engineering, National Sun Yat-sen University, Kaohsiung, Taiwan. He is currently working with the Autonomous Robotics Research Centre, Technology Innovation Institute, Abu Dhabi 51133, United Arab Emirates. This work is a part of outcome during his tenure at National Sun Yat-sen University, Kaohsiung, Taiwan. (e-mail: prasanna.raut@tii.ae).

K. Singh and C.-P. Li are with the Institute of Communications Engineering, National Sun Yat-sen University, Kaohsiung, Taiwan. (e-mail keshav.singh@mail.nsysu.edu.tw, cpli@faculty.nsysu.edu.tw).

This work was supported in part by the research grant from the Ministry of Science and Technology of Taiwan under grants MOST 109-2221-E-110 $-050-$ MY3 \& MOST 109-2222-E-110-003 and by the research grant (file number 13(22)/2020-CC\&BT) from Ministry of Electronics and Information Technology, Government of India, New Delhi. out of which 25 billion would be contributed by connections related to Internet-of-Things (IoT) which include connected cars, sensors, consumer-electronics, machines, etc. [1], [2]. It is expected that the peak data-rate and the user experience data-rate would reach to 1-20 Gbps and 10-100 Mbps, respectively [3]. Moreover, the spectral efficiency is expected to be $\times 1-\times 3$, and the connection density is expected to grow to $10 \mathrm{k}-10 \mathrm{~m}$ devices $/ \mathrm{km}^{2}$. Several technologies including ultra-dense networks, artificial intelligence (AI) empowered wireless networks, millimeter wave (mmWave), multiple-input multiple-output (MIMO) play a significant role in addressing the needs of the upcoming developments of the wireless technology [4]. For example, MIMO allows us to achieve a better spectral efficiency with the help of parallel multiple transmissions over the same bandwidth [5]. Large number of antenna deployment at the transmitting as well as receiving ends for high channel capacity is a major limitation of MIMO. This limitation can be addressed by introducing the energy efficient passive reconfigurable intelligent surfaces (RISs) which can be placed on various objects such as cars, buildings, etc. [4], [6]. The RIS technology has evolved recently as a promising technology with high potential to address the upcoming technology demands. RISs are the reconfigurable arrays of low-cost sub-wavelength sized passive elements (or meta-atoms) which can reflect the electromagnetic signals [7] and thus, they gain huge advantages over conventional relaying protocols. The electromagnetic properties of RISs can be engineered in order to achieve the adjustable magnitude and phase of the reflected signal. An RIS-assisted communication can be a cost and resource efficient technology that can support the communication in an IoT or smart industry (such as industry 4.0) environments. Considering its benefits, RIS is considered as an evolving technology for fifth generation (5G) and beyond 5G (B5G).

One of the main objectives of fifth $5 \mathrm{G}$ and B5G wireless communication systems is the energy efficiency (EE) (i.e., to reduce the power consumption without compromising the reliability performance of the system). In this aspect, energy harvesting (EH) techniques have evolved in the last few years as a green communication technology. The EH allows simultaneous wireless information and power transfer (SWIPT) in which both the energy as well as information are carried over the RF signals [8]-[10]. The EH can be classified as time-splitting (TS) EH and power-splitting (PS) EH. In the former, the EH is performed for a fraction of symbol duration, whereas the symbol is transmitted in the remaining fraction of duration using the harvested energy. Whereas, in the latter, 
a fraction of energy is harvested and remaining is used for information decoding (ID). In linear $\mathrm{EH}$, the transmit power of the device linearly depends on the input power of the energy harvester, where the practical limitations of electrical circuitry, such as sensitivity and saturation, are not considered. Therefore, in [10], a practical non-linear TS EH full-duplex (FD) relay model was proposed and the outage performance as well as the quality-of-service (QoS) were analyzed. In non-linear $\mathrm{EH}$, no energy is harvested till the input power at the harvester is less than a sensitivity value after which the harvester linearly harvests the energy till the input power reaches a saturation value. Similarly, [9] has presented a hybrid power-time splitting (PTS) EH at an FD relay and analyzed the block error rate (BLER), EE and system goodput for shortpacket communication. In [8], SWIPT in relaying has been introduced for networks which operate with fixed block length messages. Along with efficient power utilization, effective spectrum utilization is also necessary, which can be achieved by using FD relaying. This is due to the fact that, unlike halfduplex (HD) where either reception or transmission occurs at a time, FD allows reception as well as transmission at the same time over the same frequency [11]. As the RISs can be reconfigured to achieve the constructive combining of reflected signals at the receiver, it may potentially reduce the effects of channel fading and self-interference (SI) at FD receivers through boosting the desired signal.

Another important factor that contributes to the practicality and improved performance of the wireless communication system is the multiple-user scenario, which has been considered in several recent works [11]-[13]. For example, in [12] absolute (ABS) and normalized (NRM) signal-to-noise (SNR) based scheduling schemes were studied with adaptive and affordablerate transmission, and the expressions for average access time, average waiting time and average access rate were derived. Multiple user scheduling for FD two way relaying has been presented in [11], where the user pair is selected based on the max-min scheduling based on channel state information (CSI) and system state information (SSI). In [13] user selection based on channel norm and distance has been proposed for two way relaying communication along with physical network coding.

With focus on Industry 4.0 in recent years, the industrial automation has evolved as an important area of interest. The industrial automation leads to a requirement of controlling the machineries and devices by a distant controller, which makes the analysis of efficient communication involving EH, RIS and other methodologies for this purpose, becomes necessary. These applications demand high reliability and low-latency of the system, which can be improved by considering shortpacket communications [8], [9], [14]. For example, [8] and [9] have used EH-enabled relaying for short-packet communication.

\section{A. Related work}

RIS-based communication has recently been explored by the research communities. For example, a point-to-point link for a base-station to user communication with RIS has been proposed in [15], where the system rate and energy effi- ciency are optimized by varying the number of RIS metaatoms. Recently, an indoor and outdoor environment based RIS assisted communication was proposed in [16], where the system model with a single RIS has been explored for both the scenarios. A two-hop hybrid RIS and relay based communication system was presented in [17], where the RIS assists the communication between source and relay in the first hop and relay to destination in the second hop. The authors have derived tight upper-bounds for achievable rates and analyzed the optimum transmission mode. A deep learning based indoor focussing based algorithm was proposed in [18]. An RIS assisted cognitive radio communication was studied in [19], where a single secondary user co-exists with multiple primary users. Several works have considered the communication networks for IoT and industry 4.0 [8], [20][22]. For example, in [20], polarization diversity for massive MIMO system was studied for industrial environment. An overview of the holographic MIMO surfaces (HMIMOS) was presented in [23], where the hardware architecture of reconfigurable surfaces, and the opportunities and important challenges for the HMIMOS design were discussed. The RIS-assisted communication has been analyzed for several scenarios like IoT, unmanned aerial vehicles (UAV), etc. [24]-[31]. An RISenabled IoT network was considered in [24], where using the Fisher-Snedecor $F$ model, the shadowing and composite channels between a receiver-transmitter pair were analyzed. Another work that deploys RIS for IoT environment is [25], where sum mean squared error (SMSE) was minimized. The authors in [26] have introduced ambient backscatter communication assisted by RIS for short-range low-power IoT. To maximize the achievable rate in a UAV based system, an RISassisted communication for was proposed in [27].

RIS technology has been explored very recently for various wireless communication applications such as security [32][34], EH and wireless power transfer [35], [36], beamforming [4], non-orthogonal multiple access [37]-[39] and terminal positioning [40] etc. Recently, several works have also considered FD, SWIPT, ultra-reliable low-latency communications (URLLC) methods with RIS [8], [41]-[43]. The possible directions for the use of RIS technology in FD wireless communications have been discussed recently in [41]. They concluded that RISs can provide power-efficient support to FD transmission links for the devices which are out of the coverage of each other. However, the primary challenge of RIS-assisted FD communications is in the software design for the circuitry that controls the electromagnetic properties of the RIS. The URLLC constrained uplink communication for RIS-assisted system for sensor data transmission in industrial environment was presented in [42], and for mobile UAV system in [43].

Recently, several multi-user scenario involving RIS can be found in literature [44], [45]. For example, in [44] a multi-user scenario involving RIS was presented, where an energy efficiency (EE) maximization problem is addressed for optimization of RIS phase shifts and transmit power to maintain a certain quality of standard (QoS). Further, a deep-reinforcement learning based non-convex optimization algorithm for phase shifts is developed for the multiple-user 
scenario in [45].

\section{B. Motivations and contributions}

Motivated by the benefits of RIS, EH, URLLC and multiuser diversity, and the need for analyzing the communication for industrial automation, in this paper, we consider an RISassisted wireless system with non-linear $\mathrm{EH}$ for industrial applications with URLLC. We consider a data-center (DC) which communicates with multiple destination machines with the help of an FD server machine (SM) in an industrial environment. We adopt a non-linear hybrid PTS EH model to harvest energy at the SM. To the best of the authors' knowledge, none of the previous works [8], [41]-[43] have considered an RIS assisted FD-enabled hybrid PTS non-linear EH server-machine (SM) in URLLC with multiple destinations for industrial applications. The major contributions of this paper are as follows.

1) RIS allows a controlled beamforming towards the desired receiver, improving the signal strength and quality of the received signal over longer distance, thus in this work, an RIS is used to assist the communication between the DC and SM in an industrial environment.

2) Industrial applications demand for efficient power utilization. To address this aspect, the SM adopts the nonlinear hybrid PTS model for EH. For efficient spectrum utilization, the SM is considered to be operating in the FD mode of transmission.

3) Since the SM is responsible for scheduling of the destinations, we consider three scheduling schemes namely random scheduling, ABS channel-power-based (CPB) scheduling and NRM CPB scheduling schemes. Further, the end-to-end approximate closed-form expressions for the BLER and the resultant cumulative distribution function (CDF) for all the scheduling schemes are derived. Moreover, by considering the high-reliability aspects for industrial automation, short packet communication is used.

4) Finally, the performance of the system with non-linear $\mathrm{EH}$ is compared with the linear EH scenario for different number of RIS atoms. We further analyze the effect of various parameters, such as number of RIS metaatoms, positioning of RIS, non-linear EH, packet size, blocklength, fairness of scheduling schemes, etc., on the system performance. To confirm the correctness of the analysis, the Monte-Carlo simulations are used.

Rest of the paper is organized as follows. The system model and non-linear hybrid EH scheme are described in Section II, while the performance analysis is presented in Section III. The numerical results are presented and discussed in Section IV. Finally, the conclusions and future scope are mentioned in Section V. The notations used in this paper are tabulated in Table I.

\section{SySTEM MODEL}

In this paper, we consider an RIS-assisted wireless communication system in an industrial environment as shown in Fig. 1. A DC communicates with $K$ number of destinations $(D)$, but with one in the current time-slot, with the help of an RISassisted SM. The DC and $D$ are assumed to operate in HD mode, whereas SM operates in FD transmission mode. The DC comprises of one transmit antenna, whereas $D$ has one receive antenna. The FD SM consists of two antennas, one each for reception from RIS and transmission to $D$. The FD transmission at the SM results in the residual self interference (RSI) between its transmitting and receiving antennas [46]. We consider the downlink communication to the destination machines, hence the DC comprises of single antenna [15]. The $D$ is placed inside the industrial indoor environment, which receives the control signals from a distant controller placed in an outdoor environment. The RIS with large number of reflecting meta-atoms can achieve high beamforming gain as of massive MIMO with significantly low power consumption [4], assisting the communication by single-antenna possible. The SM acts as an intermediate communicating device that assists the reception of the information from the DC from outdoors and communicates it to the scheduled $D$ placed in the industrial environment. The distance between the DC and SM. i.e. $d_{s r}$, is larger as compared to the distance between SM and $k$ th $D$, i.e. $d_{r k}$, hence the signal transmitted by DC may attenuate significantly till it reaches the SM. Therefore, the reception at the SM is assisted by an RIS having $N$ metaatoms. The RIS assists reception at the SM, by forming a controlled beam towards SM and by controlling the reflection coefficient of each meta-atom. While the reflection coefficient of the $n$th meta-atom is assumed to be $\eta_{n} e^{j \theta_{n}}, h_{s i_{n}}, h_{i_{n} r}$ and $h_{r k}$ represent the channels from DC to $n$th RIS atom, $n$th RIS atom to SM and SM to $k$ th $D$, respectively. For simplicity, it is assumed that the direct channels from DC to SM, and DC to $D$ and RIS to $D$ do not exist because of a poor channel conditions [47]. The DC to SM and the SM to destination transmissions take place simultaneously. The SM is responsible for scheduling of the destinations. Furthermore, the environment from DC to SM is outdoor and that from SM to destinations is indoor industrial environment. Considering these facts and larger distance (deep fading) between RIS to destinations, the signal strength received at destinations directly from RIS is significantly small and hence can be ignored. Furthermore, We assume the channels $\left|h_{p q}\right|$ to be Nakagami- $m$ distributed with parameters $m_{p q}$ and $\Omega_{p q}$, whereas $(p q) \in\left\{\left(s i_{n}\right),\left(i_{n} r\right),(r k),(r r)\right\}$, and $h_{r r}$ denotes the RSI channel. The Nakagami- $m$ distribution is a generalized distribution and can be related to other distributions like Rayleigh, etc. by varying the value of $m_{p q}$. Recently, Nakagami- $m$ distributed channels have been explored for RIS applications [48], [49]. Based on the scheduling schemes deployed, the resultant channel between SM and the scheduled $D$ varies and is given as $h_{r d}$. Note that, for single user scenario, $(r k)=(r d)$. Therefore, the probability distribution function (PDF) and CDF respectively are given as

$$
\begin{gathered}
f_{\left|h_{p q}\right|}(x)=\frac{2 m_{p q}^{m_{p q}}}{\Gamma\left(m_{p q}\right) \Omega_{p q}^{m_{p q}}} x^{2 m_{p q}-1} \exp \left(-\frac{m_{p q}}{\Omega_{p q}} x^{2}\right) ; x>0 \\
\text { and, } F_{\left|h_{p q}\right|}(x)=\frac{\gamma\left(m_{p q}, \frac{m_{p q}}{\Omega_{p q}} x^{2}\right)}{\Gamma\left(m_{p q}\right)}
\end{gathered}
$$


TABLE I: Definitions for Notations.

\begin{tabular}{|l|l|l|}
\hline Sr. No. & Notation & \\
\hline 1 & DC & Data-center \\
\hline 2 & $D$ & Destination \\
\hline 3 & SM & Server machine \\
\hline 4 & $h_{s i_{n}}$ & Channel from DC to $n$th RIS atom \\
\hline 5 & $h_{i_{n}}$ & Channel from $n$th RIS atom to SM \\
\hline 7 & $h_{s r}$ & Channel from DC to SM \\
\hline 8 & $h_{r k}$ & Channel from SM to $k$ th $D$ \\
\hline 9 & $P_{s}$ & Transmit power of DC \\
\hline 10 & $P_{r}$ & Transmit power of SM \\
\hline 11 & $m_{p q}$ & Shape parameter for the channel indicated by the pair $p q$, where $(p q) \in\left\{\left(s i_{n}\right),\left(i_{n} r\right),(r k),(r r)\right\}$ \\
\hline 12 & $\Omega_{p q}$ & Channel power for channel indicated by the pair $p q$, where $(p q) \in\left\{\left(s i_{n}\right),\left(i_{n} r\right),(r k),(r r)\right\}$ \\
\hline 13 & $\eta_{n}$ & Reflection coefficient at each RIS atom \\
\hline 14 & $\theta_{n}$ & Phase of reflection coefficient \\
\hline 15 & $N$ & Number of RIS atoms \\
\hline 16 & $w_{r}, w_{d}$ & AWGN noise for DC to SM, SM to $D$, respectively \\
\hline 17 & $f(\cdot)$ & Probability density function (PDF) \\
\hline 18 & $F(\cdot)$ & Cumulative distribution function $(\mathrm{CDF})$ \\
\hline 19 & $M$ & Number of channels \\
\hline 20 & $\mu_{s}$ & Number of channel uses \\
\hline 21 & $K$ & Number of destinations \\
\hline
\end{tabular}

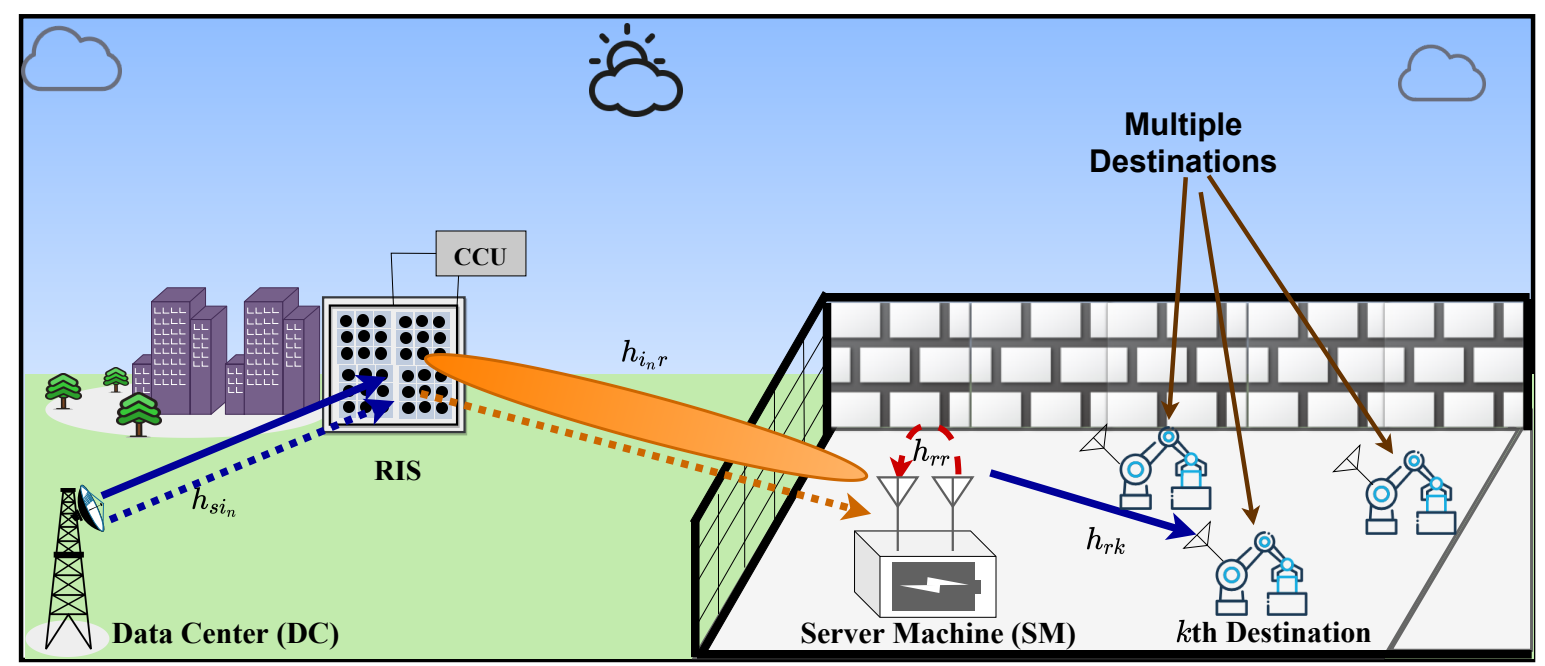

Fig. 1: Considered RIS-assisted and non-linear EH based system model for its possible industry applications.

where $m_{p q}$ is the shape parameter and $\Omega_{p q}$ is the channel power. Note that $\Omega_{p q}$ is determined using the distance dependent path-loss model. In order to efficiently use the limited amount of energy at the SM, the SM receiver considers a non-linear hybrid PTS EH.

The transmission takes place in a time-slotted fashion, such that each frame is divided into an initialization interval of $L_{i}$ length symbols and $M$ length symbols corresponding to transmission duration. The initialization interval is required for SWIPT parameter decision and blocklength allocation, whereas in the transmission duration, the DC transmits the signal, SM performs EH and the signal is received at $D$. The total duration of the frame is given as $\left(L_{i}+M\right) T_{s}$, where $T_{s}$ is the symbol duration.

\section{A. Received signal statistics}

Let DC transmits the symbol $x_{s}[l]$ to the SM in the $l$ th time slot with transmission power $P_{s}$. The signal received at the SM in the $l$ th time slot is given as

$$
y_{r}[l]=\sqrt{P_{s}} h_{s r} x_{s}[l]+\sqrt{P_{r}} h_{r r} x_{r}[l]+w_{r},
$$

where $w_{r}$ is the additive white Gaussian noise (AWGN) with $w_{r} \sim \mathcal{N}\left(0, \sigma_{r}^{2}\right), \sqrt{P_{r}} h_{r r} x_{r}[l]$ is the RSI such that the SM transmits $x_{r}[l]$ in $l$ th time-slot with power $P_{r}$. Here, $h_{s r}$ is the equivalent channel from DC to SM, considering RIS assisted reception at the SM and is given by

$$
h_{s r}=\sum_{n=1}^{N} h_{s i_{n}} \eta_{n} e^{j \theta_{n}} h_{i_{n} r} .
$$


The channel coefficients can be represented as $h_{s i_{n}}=$ $\left|h_{s i_{n}}\right| e^{j \theta_{s i_{n}}}$ and $h_{i_{n} r}=\left|h_{i_{n} r}\right| e^{j \theta_{i_{n} r}}$. As discussed previously, the beamforming is achieved by intelligently controlling the reflection coefficient. Therefore, to have optimal signal reception at SM and maximize the signal-to-interference-plus-noise ratio (SINR) of the DC to SM link, the resultant phase of $h_{s r}$ must be zero, i.e., $\theta_{n}+\theta_{s i_{n}}+\theta_{i_{n} r}=0$, or $\theta_{n}=-\left(\theta_{s i_{n}}+\theta_{i_{n} r}\right)$ [4], [50]. Therefore, the channel coefficient $h_{s r}$ for optimal SINR becomes

$$
h_{s r}=\sum_{n=1}^{N} \eta_{n}\left|h_{s i_{n}}\right|\left|h_{i_{n} r}\right| .
$$

From (5), it can be observed that $h_{s r}$ is summation of large number of random variables, therefore, according to central limit theorem (CLT), $\left|h_{s r}\right|$ follows Gaussian distribution such that $\left|h_{s r}\right| \sim \mathcal{N}\left(\mu_{h}, \sigma_{h}^{2}\right)$, where the values of $\mu_{h}$ and $\sigma_{h}^{2}$ are given by

$$
\begin{aligned}
\mu_{h}= & \eta_{n} \sum_{n=1}^{N}\left(\frac{\Omega_{s i_{n}} \Omega_{i_{n} r}}{m_{s i_{n}} m_{i_{n} r}}\right)^{\frac{1}{2}} \frac{\Gamma\left(m_{s i_{n}}+\frac{1}{2}\right) \Gamma\left(m_{i_{n} r}+\frac{1}{2}\right)}{\Gamma\left(m_{s i_{n}}\right) \Gamma\left(m_{i_{n} r}\right)}, \\
\sigma_{h}^{2}= & \eta_{n}^{2} \sum_{n=1}^{M}\left[\Omega_{s i_{n}} \Omega_{i_{n} r}\right. \\
& \left.-\left(\left(\frac{\Omega_{s i_{n}} \Omega_{i_{n} r}}{m_{s i_{n}} m_{i_{n} r}}\right)^{\frac{1}{2}} \frac{\Gamma\left(m_{s i_{n}}+\frac{1}{2}\right) \Gamma\left(m_{i_{n} r}+\frac{1}{2}\right)}{\Gamma\left(m_{s i_{n}}\right) \Gamma\left(m_{i_{n} r}\right)}\right)^{2}\right] .
\end{aligned}
$$

The PDF and CDF of $\left|h_{s r}\right|$ are respectively given as

$$
\begin{aligned}
& f_{\left|h_{s r}\right|}(x)=\frac{\alpha}{\sqrt{2 \pi \sigma_{h}^{2}}} \exp \left(-\frac{\left(x-\mu_{h}\right)^{2}}{2 \sigma_{h}^{2}}\right) ; x>0, \\
& F_{\left|h_{s r}\right|}(x)=1-\alpha Q\left(\frac{x-\mu_{h}}{\sigma_{h}}\right),
\end{aligned}
$$

where $\alpha$ is the normalization factor defined as $\alpha=\left[Q\left(\frac{\mu_{h}}{\sigma_{h}}\right)\right]^{-1}$ and $Q(\cdot)$ is the standard $Q$-function defined as $Q(x)=$ $\frac{1}{\sqrt{2 \pi}} \int_{x}^{\infty} e^{-t^{2} / 2} d t$. The signal received at scheduled $D$ in $l$ th time-slot is given as

$$
y_{d}[l]=\sqrt{P_{r}} h_{r d} x_{r}[l]+w_{d},
$$

where $w_{d} \sim \mathcal{N}\left(0, \sigma_{d}^{2}\right)$ is the AWGN with variance $\sigma_{d}^{2}$.

\section{B. CSI estimation and central control unit}

Recently, several works can be found that address the CSI estimation for RIS based communication systems [51]-[54]. We assume that the DC, SM and RIS are fully coordinated with the help of a central control unit (CCU). The CCU assists in gathering the CSI, such that the CSI is completely known at the RIS for tuning the phases of reflection coefficients. The CSI estimation is performed with techniques such as PARAllel FACtor (PARAFAC) decomposition based algorithms [51].

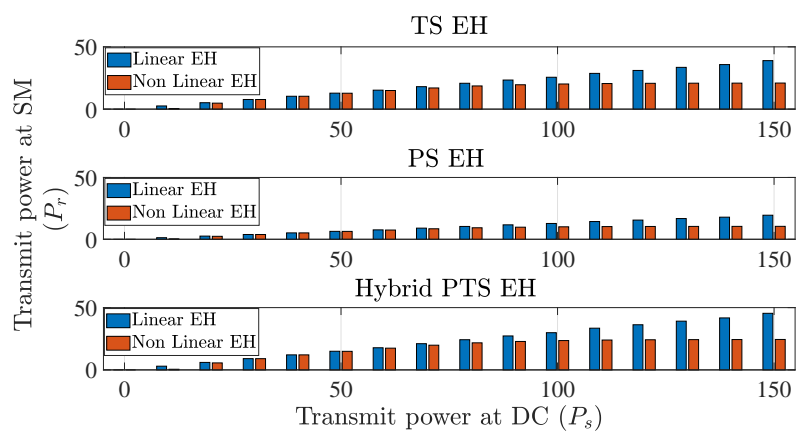

Fig. 2: $P_{r}$ vs $P_{s}$ for PS, TS and hybrid PTS EH.

\section{Non-linear hybrid power-time splitting EH}

The SM adopts a non-linear hybrid PTS EH. The traditional linear EH techniques lack the practicality of the energy harvester, that would not perform EH till the input power is less than a sensitivity value $P_{\min }$ as well as saturation $P_{\max }$ [9], [10]. In order to achieve the efficient EH, we adopt a hybrid PTS EH [9]. In PTS EH, the transmission phase $M$ is divided into three phases, $v, n_{1}$ and $n_{2}$. In the $v$, the SM performs EH using the TS protocol, in interval $n_{1}$, the SM performs EH using the PS protocol with PS ratio $\rho$ and ID with $(1-\rho)$ fraction, where $\rho \in[0,1]$, and for remaining $n_{2}$ interval, the SM performs the transmission from SM to desired $D$. The energy harvested by TS and PTS protocol is given as $\eta v T_{S} P_{i n}$ and $\eta \rho n_{1} T_{S} P_{i n}$ respectively, where $\eta$ is the power efficiency factor and the input power is given as $P_{i n}=\left|h_{s r}\right|^{2} P_{s}$. Therefore, the net energy harvested is given as $E_{\mathrm{PTS}}=\eta\left(v T_{s}+\rho n_{1} T_{s}\right) P_{i n}$. The transmit power of SM is given as

$$
P_{r}= \begin{cases}0, & P_{\text {in }}<P_{\text {min }}, \\ \beta P_{s}\left|h_{s r}\right|^{2}, & P_{\text {min }} \leq P_{\text {in }} \leq P_{\text {max }}, \\ \beta P_{\text {max }}, & P_{\text {in }}>P_{\text {max }},\end{cases}
$$

where, $\beta=\frac{\eta\left(v T_{s}+\rho n_{1} T_{s}\right)}{n_{2} T_{s}}$. In order to analyze the efficiency of the EH protocols, we plot the $P_{r}$ vs the $P_{s}$ for TS, PS, and hybrid PTS EH is as shown in Fig. 2. It can be observed that highest EH is observed in case of Hybrid PTS EH as compared to TS EH and PS EH.

\section{Performance Analysis}

Using (3) and (10), the SINR for DC to SM link and SNR for the SM to $D$ link are given as $\Upsilon_{s r}$ and $\Upsilon_{r d}$, respectively, as

$$
\begin{aligned}
& \Upsilon_{s r}=\frac{P_{s}\left|h_{s r}\right|^{2}}{P_{r}\left|h_{r r}\right|^{2}+\sigma_{r}^{2}}, \\
& \Upsilon_{r d}=\frac{P_{r}\left|h_{r d}\right|^{2}}{\sigma_{d}^{2}} .
\end{aligned}
$$

Note that $\left|h_{r d}\right|$ is the equivalent channel between the SM and the selected $D$. Based on the values of $P_{r}$ as in (11), $\Upsilon_{s r}$ and $\Upsilon_{r d}$ are written as [10]

$$
\Upsilon_{s r}= \begin{cases}\frac{P_{s}\left|h_{s r}\right|^{2}}{\sigma_{r}^{2}}, & P_{i n}<P_{\text {min }}, \\ \frac{1}{\beta\left|h_{r r}\right|^{2}}, & P_{\text {min }} \leq P_{\text {in }} \leq P_{\text {max }}, \\ \frac{P_{s}\left|h_{s r}\right|^{2}}{\beta P_{\text {max }}\left|h_{r r}\right|^{2}}, & P_{\text {in }}>P_{\text {max }},\end{cases}
$$


and

$$
\Upsilon_{r d}= \begin{cases}0, & P_{i n}<P_{\text {min }}, \\ \frac{\beta P_{s}\left|h_{s r}\right|^{2}\left|h_{r d}\right|^{2}}{\sigma_{d}^{2}}, & P_{\text {min }} \leq P_{i n} \leq P_{\text {max }} \\ \frac{\beta P_{\max }\left|h_{r d}\right|^{2}}{\sigma_{d}^{2}}, & P_{i n}>P_{\text {max }},\end{cases}
$$

respectively.

The $\left|h_{p q}\right|^{2}$ forms the gamma distribution with parameters $m_{p q}$ and $\Omega_{p q}$ where $(p q) \in\left\{\left(s i_{n}\right),\left(i_{n} r\right),(r k),(r r)\right\}$.

The PDF and CDF for $\left|h_{p q}\right|^{2}$ are respectively given as

$$
f_{\left|h_{p q}\right|^{2}}(x)=\frac{m_{p q}^{m_{p q}}}{\Gamma\left(m_{p q}\right) \Omega_{p q}^{m_{p q}}} x^{m_{p q}-1} \exp \left(-\frac{m_{p q}}{\Omega_{p q}} x\right),
$$

and

$$
F_{\left|h_{p q}\right|^{2}}(x)=\frac{\gamma\left(m_{p q}, \frac{m_{p q}}{\Omega_{p q}} x\right)}{\Gamma\left(m_{p q}\right)} .
$$

Similarly, the CDF and PDF of $\left|h_{s r}\right|^{2}$ are respectively given as [50]

$$
\begin{aligned}
F_{\left|h_{s r}\right|^{2}}(x) & =1-\alpha Q\left(\frac{\sqrt{x}-\mu_{h}}{\sigma_{h}}\right), \\
f_{\left|h_{s r}\right|^{2}}(x) & =\frac{\alpha}{2 \sqrt{2 \pi \sigma_{h}^{2} x}} \exp \left(\frac{\left(\sqrt{x}-\mu_{h}\right)^{2}}{2 \sigma_{h}^{2}}\right) .
\end{aligned}
$$

\section{A. CDF of end-to-end SINR}

For the BLER analysis, first we find the CDF of the end-toend SINR. The end-to-end SINR is mathematically calculated as

$$
\Upsilon=\min \left(\Upsilon_{s r}, \Upsilon_{r d}\right)
$$

The end-to-end SINR's CDF $\left(F_{\Upsilon}\left(\Upsilon_{t h}\right)\right)$ is given as

$$
\begin{aligned}
F_{\Upsilon}\left(\Upsilon_{t h}\right) & =P\left(\min \left(\Upsilon_{s r}, \Upsilon_{r d}\right)<\Upsilon_{t h}\right), \\
& =1-P\left(\Upsilon_{s r} \geq \Upsilon_{t h} \cap \Upsilon_{r d} \geq \Upsilon_{t h}\right), \\
& =1-P\left(\left(P_{i n} \geq P_{\min }\right) \cap\left(\Upsilon_{s r}>\Upsilon_{t h}\right) \cap\left(\Upsilon_{r d}>\Upsilon_{t h}\right)\right), \\
& =1-I_{1}-I_{2},
\end{aligned}
$$

where $I_{1}$ and $I_{2}$ are respectively defined as

$$
\begin{aligned}
I_{1} & =P\left(P_{\text {min }} \leq P_{\text {in }} \leq P_{\text {max }} \cap \Upsilon_{s r}>\Upsilon_{t h} \cap \Upsilon_{r d}>\Upsilon_{t h}\right), \\
& = \begin{cases}0, & \left|h_{r d}\right|^{2}<\frac{D}{C}, \\
P\left(\left|h_{r r}\right|^{2}<A\right) P\left(\frac{D}{\left|h_{r d}\right|^{2}}<\left|h_{s r}\right|^{2} \leq C\right), & \frac{D}{C}<\left|h_{r d}\right|^{2}<\frac{D}{B}, \\
P\left(\left|h_{r r}\right|^{2}<A\right) P\left(B<\left|h_{s r}\right|^{2} \leq C\right), & \left|h_{r d}\right|^{2}>\frac{D}{B},\end{cases}
\end{aligned}
$$

and

$$
\begin{aligned}
I_{2} & =P\left(P_{i n} \geq P_{\max } \cap \Upsilon_{s r}>\Upsilon_{t h} \cap \Upsilon_{r d}>\Upsilon_{t h}\right), \\
& = \begin{cases}P\left(\left|h_{r d}\right|^{2}>E\right) P\left(\left|h_{s r}\right|^{2}>C\right), & \left|h_{r r}\right|^{2}<A, \\
P\left(\left|h_{r d}\right|^{2}>E\right) P\left(\left|h_{s r}\right|^{2}>\frac{C}{A}\left|h_{r r}\right|^{2}\right), & \left|h_{r r}\right|^{2}>A,\end{cases}
\end{aligned}
$$

where $A=\frac{1}{\beta \Upsilon_{t h}}, B=\frac{P_{\min }}{P_{s}}, C=\frac{P_{\max }}{P_{s s}}, D=\frac{\sigma_{d}^{2} \Upsilon_{t h}}{\beta P_{s}}$ and $E=\frac{\sigma_{d}^{2} \Upsilon_{t h}}{\beta P_{\max }}$. Further, we derive the approximate closed-form expressions for $I_{1}$ and $I_{2}$ as

$$
\begin{gathered}
I_{1}=\int_{0}^{A} f_{\left|h_{r r}\right|^{2}}(x) d x \int_{\frac{D}{C}}^{\frac{D}{B}}\left(f_{\left|h_{r d}\right|^{2}}(x) \int_{\frac{D}{x}}^{C} f_{\left|h_{s r}\right|^{2}}(y) d y\right) d x \\
+\int_{0}^{A} f_{\left|h_{r r}\right|^{2}}(x) d x \int_{B}^{C} f_{\left|h_{s r}\right|^{2}}(x) d x \int_{\frac{D}{B}}^{\infty} f_{\left|h_{r d}\right|^{2}}(x) d x \\
I_{1}=F_{\left|h_{r r}\right|^{2}}(A) \times\left[I_{3}+\left(F_{\left|h_{s r}\right|^{2}}(C)-F_{\left|h_{s r}\right|^{2}}(B)\right)\right. \\
\left.\times\left(1-F_{\left|h_{r d}\right|^{2}}\left(\frac{D}{B}\right)\right)\right]
\end{gathered}
$$

where

$$
\begin{aligned}
I_{3} & =\int_{\frac{D}{C}}^{\frac{D}{B}}\left(f_{\left|h_{r d}\right|^{2}}(x) \int_{\frac{D}{x}}^{C} f_{\left|h_{s r}\right|^{2}}(y) d y\right) d x, \\
& =\int_{\frac{D}{C}}^{\frac{D}{B}} f_{\left|h_{r d}\right|^{2}}(x)\left[F_{\left|h_{s r}\right|^{2}}(C)-F_{\left|h_{s r}\right|^{2}}\left(\frac{D}{x}\right)\right] d x, \\
& =F_{\left|h_{s r}\right|^{2}}(C)\left(F_{\left|h_{r d}\right|^{2}}\left(\frac{D}{B}\right)-F_{\left|h_{r d}\right|^{2}}\left(\frac{D}{C}\right)\right)-I_{4} .
\end{aligned}
$$

The term $I_{4}$ in (26) is defined as

$$
I_{4}=\int_{\frac{D}{C}}^{\frac{D}{B}} f_{\left|h_{r d}\right|^{2}}(x) F_{\left|h_{s r}\right|^{2}}\left(\frac{D}{x}\right) d x .
$$

We use the Gaussian-Chebyshev-Quadrature (GCQ) method to approximate (27) [55, eq. 25.4.30] as

$$
I_{4}=\frac{\frac{D}{B}+\frac{D}{C}}{2} \sum_{g=1}^{G} \frac{\pi}{G} \sqrt{1-\phi_{g}^{2}} f_{\left|h_{r d}\right|^{2}}\left(z_{1}\right) F_{\left|h_{s r}\right|^{2}}\left(\frac{D}{z_{1}}\right)+R_{G},
$$

where $\phi_{g}=\cos \left(\frac{2 g-1}{2 G} \pi\right), z_{1}=\left(\frac{\frac{D}{B}-\frac{D}{C}}{2}\right) \phi_{g}+\left(\frac{\frac{D}{B}+\frac{D}{C}}{2}\right), G$ is the complexity-accuracy trade-off factor and $R_{G}$ is the error term that can be neglected at sufficiently higher value of $G$. The final approximate closed-form expression for $I_{1}$ is given in (29), shown on the top of the next page.

Similarly, the expression for $I_{2}$ is given as

$$
\begin{aligned}
I_{2}= & \int_{E}^{\infty} f_{\left|h_{s r}\right|^{2}}(x) d x \int_{A}^{\infty}\left(f_{\left|h_{r r}\right|^{2}}(x) \int_{\frac{C}{A} x}^{\infty} f_{\left|h_{s r}\right|^{2}}(y) d y\right) d x \\
& +\int_{E}^{\infty} f_{\left|h_{s r}\right|^{2}}(x) d x \int_{C}^{\infty} f_{\left|h_{s r}\right|^{2}}(x) d x \int_{0}^{A} f_{\left|h_{r r}\right|^{2}}(x) d x, \\
I_{2}= & \left(1-F_{\left|h_{r d}\right|^{2}}(E)\right) \times\left[I_{5}+\left(1-F_{\left|h_{s r}\right|^{2}}(C)\right) F_{\left|h_{r r}\right|^{2}}(A)\right],
\end{aligned}
$$

where

$$
\begin{aligned}
I_{5} & =\int_{A}^{\infty}\left(f_{\left|h_{r r}\right|^{2}}(x) \int_{\frac{C}{A} x}^{\infty} f_{\left|h_{s r}\right|^{2}}(y) d y\right) d x, \\
& =1-\int_{0}^{A} f_{\left|h_{r r}\right|^{2}}(x)\left(1-\int_{0}^{\frac{C}{A} x} f_{\left|h_{s r}\right|^{2}}(y) d y\right) d x, \\
& =1-F_{\left|h_{r r}\right|^{2}}(A)+I_{6} .
\end{aligned}
$$




$$
\begin{aligned}
& I_{1}=F_{\left|h_{r r}\right|^{2}}(A) \times\left[F_{\left|h_{s r}\right|^{2}}(C)\left(F_{\left|h_{r d}\right|^{2}}\left(\frac{D}{B}\right)-F_{\left|h_{r d}\right|^{2}}\left(\frac{D}{C}\right)\right)-\left(\frac{\frac{D}{B}+\frac{D}{C}}{2} \sum_{g=1}^{G} \frac{\pi}{G} \sqrt{1-\phi_{g}^{2}} f_{\left|h_{r d}\right|^{2}}\left(z_{1}\right) F_{\left|h_{s r}\right|^{2}}\left(\frac{D}{z_{1}}\right)+R_{G}\right)\right. \\
& \left.+\left(F_{\left|h_{s r}\right|^{2}}(C)-F_{\left|h_{s r}\right|^{2}}(B)\right) \times\left(1-F_{\left|h_{r d}\right|^{2}}\left(\frac{D}{B}\right)\right)\right] .
\end{aligned}
$$

Here $I_{6}$ in (32) is defined as

$$
\begin{aligned}
I_{6} & =\int_{0}^{A} f_{\left|h_{r r}\right|^{2}}(x)\left(\int_{0}^{\frac{C}{A} x} f_{\left|h_{s r}\right|^{2}}(y) d y\right) d x, \\
& =\int_{0}^{A} f_{\left|h_{r r}\right|^{2}}(x) F_{\left|h_{s r}\right|^{2}}\left(\frac{C}{A} x\right) d x .
\end{aligned}
$$

Substituting $x=A y$ in (33), we get

$$
I_{6}=A \int_{0}^{1} f_{\left|h_{r r}\right|^{2}}(A y) F_{\left|h_{s r}\right|^{2}}(C y) d y .
$$

$I_{6}$ in (34) is approximated using the GCQ method as [55]

$$
I_{6}=A \sum_{l_{1}=1}^{L} \frac{\pi}{2 L} \sqrt{1-\phi_{l_{1}}^{2}} f_{\left|h_{r r}\right|^{2}}\left(A z_{2}\right) F_{\left|h_{s r}\right|^{2}}\left(C z_{2}\right)+R_{L},
$$

where $\phi_{l_{1}}=\cos \left(\frac{2 l_{1}-1}{2 L} \pi\right), z_{2}=\left(\frac{\phi_{l_{1}}+1}{2}\right), L$ is the complexityaccuracy trade-off factor and $R_{L}$ is the error term that can be neglected at sufficiently higher values of $L$. The final approximate closed-form expression for $I_{2}$ is given in (36), shown on the bottom of this page. Finally, the CDF can be obtained by substituting (29) and (36) in (21).

\section{B. Multi-destination Scheduling}

In order to transmit the data to the destination, the scheduling is performed at the SM. Three scheduling schemes, namely RND scheduling, ABS CPB scheduling and NRM $\mathrm{CPB}$ scheduling are considered in this work. As the destination selection is done at the SM the channel from SM to $D$ would be affected and based on the scheduling scheme, $F_{\left|h_{r d}\right|^{2}}$ and $f_{\left|h_{r d}\right|^{2}}$ in (29) and (36) would change.

1) Random (RND) Scheduling: In RND scheduling, the SM randomly selects a destination for the data transmission. The probability that a destination is selected randomly is $\frac{1}{K}$. The CDF and PDF of $\left|h_{r d}\right|^{2}$ are respectively given as

$$
\begin{aligned}
& F_{\left|h_{r d}\right|^{2}}(x)=\frac{1}{K} \sum_{k=1}^{K} F_{\left|h_{r k}\right|^{2}}(x), \\
& f_{\left|h_{r d}\right|^{2}}(x)=\frac{1}{K} \sum_{k=1}^{K} f_{\left|h_{r k}\right|^{2}}(x) .
\end{aligned}
$$

The access probability of a user for RND scheduling scheme is given as

$$
P_{k}=\frac{1}{K} .
$$

2) Absolute ( $A B S$ ) channel-power-based ( $C P B)$ Scheduling: The destination with maximum channel power is selected in ABS CPB scheduling scheme. Therefore, the CDF for $\left|h_{r d}\right|^{2}$, when $k$ th destination is selected, is given as

$$
\begin{aligned}
F_{\left|h_{r d}\right|^{2}}(x)= & \sum_{k=1}^{K} \operatorname{Pr}\left(\left|h_{r k}\right|^{2}<x \cap\left|h_{r j}\right|^{2}<\left.h_{r k}\right|^{2}\right), \\
\forall j \in\{1,2, \ldots, K\} \text { and } j \neq k, & \sum_{k=1}^{K} \int_{0}^{x} f_{\left|h_{r k}\right|^{2}}(y) \prod_{\substack{j=1 \\
j \neq k}}^{K} F_{\left|h_{r j}\right|^{2}}(y) d y .
\end{aligned}
$$

Similarly, the PDF is given as

$$
f_{\left|h_{r d}\right|^{2}}(x)=\sum_{k=1}^{K}\left(f_{\left|h_{r k}\right|^{2}}(x) \prod_{\substack{j=1 \\ j \neq k}}^{K} F_{\left|h_{r j}\right|^{2}}(x)\right) .
$$

The probability that the $k$ th destination is accessed for ABS $\mathrm{CPB}$ scheduling scheme is given as

$$
\begin{aligned}
P_{k} & =\operatorname{Pr}\left(\left|h_{r k}\right|^{2}>\left|h_{r j}\right|^{2}\right), \forall j \in\{1,2, \ldots, K\} \text { and } j \neq k, \\
& =\int_{0}^{\infty} f_{\left|h_{r k}\right|^{2}}(x) \prod_{\substack{j=1 \\
j \neq k}}^{K} \int_{0}^{x} f_{\left|h_{r j}\right|^{2}}(y) d y d x, \\
& =\int_{0}^{\infty} f_{\left|h_{r k}\right|^{2}}(x) \prod_{\substack{j=1 \\
j \neq k}}^{K} F_{\left|h_{r j}\right|^{2}}(x) d x .
\end{aligned}
$$

Substituting the PDF and CDF of $\left|h_{r k}\right|^{2}$ and $\left|h_{r j}\right|^{2}$ as in (16) and (17) respectively and substituting $\exp \left(-\frac{m_{r k}}{\Omega_{r k}} x\right)=t$ in (42), we have

$$
P_{k}=\int_{0}^{1} b_{k}(\ln (t))^{m_{r k}-1} \prod_{\substack{j=1 \\ j \neq k}}^{K} \frac{\gamma\left(m_{r j},-a_{j} \ln (t)\right)}{\Gamma\left(m_{r j}\right)}
$$

$$
\begin{aligned}
I_{2}=\left(1-F_{\left|h_{r d}\right|^{2}}(E)\right) \times\left[1-F_{\left|h_{r r}\right|^{2}}(A)\right. & +\left(A \sum_{l=1}^{L} \frac{\pi}{2 L} \sqrt{1-\phi_{l}^{2}} f_{\left|h_{r r}\right|^{2}}\left(A z_{2}\right) F_{\left|h_{s r}\right|^{2}}\left(C z_{2}\right)+R_{L}\right) \\
& \left.+\left(1-F_{\left|h_{s r}\right|^{2}}(C)\right) F_{\left|h_{r r}\right|^{2}}(A)\right] .
\end{aligned}
$$


where, $b_{k}=\frac{(-1)^{m_{r k}-1}}{\Gamma\left(m_{r k}\right)}$ and $a_{j}=\frac{m_{r j}}{m_{r k}} \frac{\Omega_{r k}}{\Omega_{r j}}$. The approximate closed-form expression can be obtained using the GCQ method [55, eq. 25.4.30]

$$
\begin{aligned}
P_{k}=b_{k} \frac{\pi}{2 \widehat{G}} \sum_{\widehat{g}=1}^{\widehat{G}} \sqrt{1-\phi_{\widehat{g}}^{2}}\left(\ln \left(z_{g}\right)\right)^{m_{r k}-1} \\
\times \prod_{\substack{j=1 \\
j \neq k}}^{K} \frac{\gamma\left(m_{r j},-a_{j} \ln \left(z_{g}\right)\right)}{\Gamma\left(m_{r j}\right)}+R_{\widehat{G}},
\end{aligned}
$$

where $\phi_{\widehat{g}}=\cos \left(\frac{2 \widehat{g}-1}{2 \widehat{G}} \pi\right), z_{g}=\left(\frac{\phi_{\widehat{g}}+1}{2}\right), \widehat{G}$ is the complexityaccuracy trade-off factor and $R_{\widehat{G}}$ is the error term that can be neglected at sufficiently higher value of $\widehat{G}$.

3) Normalized (NRM) channel-power-based (CPB) Scheduling: Since ABS CPB scheduling scheme always selects the destination with maximum channel power, it is an unfair scheduling scheme. To address this, we use the NRM CPB scheduling scheme, where the destination with maximum normalized channel power is selected [12]. The CDF of $\left|h_{r d}\right|^{2}$, when $k$ th destination is selected, is given as

$$
\begin{aligned}
F_{\left|h_{r d}\right|^{2}}(x) & =\sum_{k=1}^{K} \operatorname{Pr}\left(\left|h_{r k}\right|^{2}<x \cap \frac{\left|h_{r j}\right|^{2}}{\Omega_{r j}}<\frac{\left|h_{r k}\right|^{2}}{\Omega_{r k}}\right), \\
= & \sum_{k=1}^{K} \operatorname{Pr}\left(\left|h_{r k}\right|^{2}<x \cap\left|h_{r j}\right|^{2}<\left|h_{r k}\right|^{2} \frac{\Omega_{r j}}{\Omega_{r k}}\right), \\
\forall j \in\{1,2, \ldots, K\} \text { and } j \neq k, & \\
= & \sum_{k=1}^{K} \int_{0}^{x} f_{\left|h_{r k}\right|^{2}}(y) \prod_{\substack{j=1 \\
j \neq k}}^{K} F_{\left|h_{r j}\right|^{2}}\left(\frac{\Omega_{r j}}{\Omega_{r k}} y\right) d y .
\end{aligned}
$$

Similarly, the PDF is given as

$$
f_{\left|h_{r d}\right|^{2}}(x)=\sum_{k=1}^{K}\left(f_{\left|h_{r k}\right|^{2}}(x) \prod_{\substack{j=1 \\ j \neq k}}^{K} F_{\left|h_{r j}\right|^{2}}\left(\frac{\Omega_{r j}}{\Omega_{r k}} x\right)\right) .
$$

The access probability of the $k$ th destination for the NRM $\mathrm{CPB}$ scheduling scheme is given as

$$
\begin{aligned}
P_{k} & =\operatorname{Pr}\left(\frac{\left|h_{r k}\right|^{2}}{\Omega_{r k}}>\frac{\left|h_{r j}\right|^{2}}{\Omega_{r j}}\right), \\
& =\operatorname{Pr}\left(\left|h_{r k}\right|^{2}>\left|h_{r j}\right|^{2} \frac{\Omega_{r k}}{\Omega_{r j}}\right), \forall j \in\{1,2, \ldots, K\} \text { and } j \neq k \\
& =\int_{0}^{\infty} f_{\left|h_{r k}\right|^{2}}(x) \prod_{\substack{j=1 \\
j \neq k}}^{K} \int_{0}^{x \frac{\Omega_{r k}}{\Omega_{r j}}} f_{\left|h_{r j}\right|^{2}}(y) d y d x \\
& =\int_{0}^{\infty} f_{\left|h_{r k}\right|^{2}}(x) \prod_{\substack{j=1 \\
j \neq k}}^{K} F_{\left|h_{r j}\right|^{2}}\left(\frac{\Omega_{r k}}{\Omega_{r j}} x\right) d x
\end{aligned}
$$

By substituting the PDF and CDF of $\left|h_{r k}\right|^{2}$ and $\left|h_{r j}\right|^{2}$ as in (16) and (17) respectively and substituting $\exp \left(-\frac{m_{r k}}{\Omega_{r k}} x\right)=t$ in (47), we get

$$
P_{k}=\int_{0}^{1} b_{k}(\ln (t))^{m_{r k}-1} \prod_{\substack{j=1 \\ j \neq k}}^{K} \frac{\gamma\left(m_{r j},-c_{j} \ln (t)\right)}{\Gamma\left(m_{r j}\right)},
$$

where $c_{j}=\frac{m_{r j}}{m_{r k}}$. The approximate closed-form expression can be obtained by using the GCQ method [55, eq. 25.4.30]

$$
\begin{aligned}
& P_{k}=b_{k} \frac{\pi}{2 \widehat{L}} \sum_{\widehat{l}=1}^{\widehat{L}} \sqrt{1-\phi_{\widehat{l}}^{2}}\left(\ln \left(z_{l}\right)\right)^{m_{r k}-1} \\
& \times \prod_{\substack{j=1 \\
j \neq k}}^{K} \frac{\gamma\left(m_{r j},-c_{j} \ln \left(z_{l}\right)\right)}{\Gamma\left(m_{r j}\right)}+R_{\widehat{L}},
\end{aligned}
$$

where $\phi_{\widehat{l}}=\cos \left(\frac{2 \widehat{l}-1}{2 \widehat{L}} \pi\right), z_{l}=\left(\frac{\phi_{\widehat{l}}+1}{2}\right), \widehat{L}$ is the complexityaccuracy trade-off factor and $R_{\widehat{L}}$ is the error term that can be neglected at sufficiently higher value of $\widehat{L}$.

\section{Fairness Analysis}

The fairness of a scheduling scheme indicates how unbiased the scheme is while selection of the destination. The selffairness for the $k$ th $D$ and the average fairness of the system are respectively given as [12]

$$
\begin{aligned}
\varsigma_{k} & =-\frac{\log \left(P_{k}\right)}{\log (K)}, \\
\bar{\varsigma}_{k} & =-\sum_{k=1}^{K} P_{k} \varsigma_{k}=-\sum_{k=1}^{K} P_{k} \frac{\log \left(P_{k}\right)}{\log (K)},
\end{aligned}
$$

where $P_{k}$ is the access probability which can be obtained as in (39), (44) and (49) for RND, ABS CPB and NRM CPB scheduling schemes, respectively.

\section{Block Error Rate}

Considering the $\epsilon_{s d}$ as the overall BLER of the considered an RIS-assisted wireless network. It is assumed that the DC transmits $b_{s}$ number of bits over $M$ channels. For $\mu_{s}$ number of channels uses, the coding rate (bits/channel uses) is given as $r_{s d}=\frac{b_{s}}{\mu_{s}}$. Therefore, the overall BLER is

$$
\epsilon_{s d}\left(r_{s d}\right) \approx \mathbb{E}\left\{Q\left(\frac{C(\Upsilon)-r_{s d}}{\sqrt{V(\Upsilon) / \mu_{s}}}\right)\right\},
$$

where $C(\Upsilon)=\log _{2}(1+\Upsilon)$ is the Shannon capacity and $V(\Upsilon)=\left(1-(1+\Upsilon)^{-2}\right)\left(\log _{2} e\right)^{2}$ is the channel dispersion that measures the stochastic channel variability relative to a deterministic channel with same channel capacity.

Due to mathematical intractability of the $\epsilon_{s d}\left(r_{s d}\right)$ to obtain a closed-form expression, we adopt the linear approximation of the $\Xi=Q\left(\frac{C(\Upsilon)-r_{s d}}{\sqrt{V(\Upsilon) / \mu_{s}}}\right)$ as [56]

$$
\Xi= \begin{cases}1, & \Upsilon \leq \zeta, \\ 0.5-\vartheta \sqrt{\mu_{s}}(\Upsilon-\theta), & \zeta<\Upsilon<\xi, \\ 0, & \Upsilon \geq \xi,\end{cases}
$$


TABLE II: Simulation Parameters

\begin{tabular}{|c|c|c|}
\hline Notation & Definition & Value \\
\hline$d_{s i}$ & Distance from DC to RIS & $95 \mathrm{~m}$ \\
\hline$d_{\text {ir }}$ & Distance from RIS to SM & $95 \mathrm{~m}$ \\
\hline$d_{s r}$ & Distance from DC to SM & $150 \mathrm{~m}$ \\
\hline$d_{r d}$ & Distance from SM to $D$ & $20 \mathrm{~m}$ \\
\hline$T_{s}$ & Symbol duration & $1 \mu \mathrm{s}$ \\
\hline$N$ & Number of RIS & 64 \\
\hline$M$ & Number of channels & 1000 \\
\hline$v$ & Number of intervals for TS EH & 50 \\
\hline$n_{1}$ & Number of intervals for PS EH & 650 \\
\hline$n_{2}$ & $\begin{array}{l}\text { Number of intervals for } \\
\text { transmission from SM to } D\end{array}$ & 300 \\
\hline$\eta_{n}$ & Reflection coefficient & 0.2 \\
\hline$\rho$ & Power splitting factor & 0.6 \\
\hline$\eta$ & Power efficiency & 0.7 \\
\hline$P_{\min }$ & Sensitivity & $0.5 \mathrm{~W}$ \\
\hline$P_{\max }$ & Saturation & $9 \mathrm{~W}$ \\
\hline$\Omega_{r r}$ & RSI Power & $-10 \mathrm{~dB}$ \\
\hline$m_{r r}$ & Shape parameter for RSI channel & 3 \\
\hline $\begin{array}{l}m_{s i_{n}} \\
m_{i_{n} r} \\
m_{r k}\end{array}$ & Shape parameters (except RSI) & 1 \\
\hline
\end{tabular}

where $\vartheta=\frac{1}{2 \pi \sqrt{2^{2 r_{s d}-1}}}, \theta=2^{r_{s d}}-1, \zeta=\theta-\frac{1}{2 \vartheta \sqrt{\mu_{s}}}$ and $\xi=\theta+\frac{1}{2 \vartheta \sqrt{\mu_{s}}}$. The final BLER is obtained by averaging $\Xi$ with respect to the PDF of $\Upsilon$. Therefore, we get

$$
\begin{gathered}
\epsilon_{s d}\left(r_{s d}\right) \approx \int_{0}^{\infty} \Xi(x) f_{\Upsilon}(x) d x \approx\left[\Xi(x) F_{\Upsilon}(x)\right]_{0}^{\infty} \\
-\int_{0}^{\infty} F_{\Upsilon}(x) d \Xi(x),
\end{gathered}
$$

where $F_{\Upsilon}(x)$ and $f_{\Upsilon}(x)$ are the CDF and PDF of $\Upsilon$ respectively. Therefore, we have

$$
\epsilon_{s d}\left(r_{s d}\right)=\vartheta \sqrt{\mu_{s}} \int_{\zeta}^{\xi} F_{\Upsilon}(x) d x
$$

In order to obtain the final expression of $\epsilon_{s d}$, we apply the GCQ method [55, eq. 25.4.30]. We have

$$
\epsilon_{s d}\left(r_{s d}\right)=\vartheta \sqrt{\mu_{s}}\left(\frac{\xi+\zeta}{2}\right) \sum_{p=1}^{P} \frac{\pi}{P} \sqrt{1-\phi_{p}^{2}} F_{\Upsilon}\left(z_{3}\right)+R_{P}
$$

where $\phi_{p}=\cos \left(\frac{2 p-1}{2 P} \pi\right), z_{3}=\left(\frac{\xi-\zeta}{2}\right) \phi_{p}+\left(\frac{\xi+\zeta}{2}\right), P$ is the complexity-accuracy trade-off factor and $R_{P}$ is the error term, being neglected for significantly higher value of $P$.

\section{Numerical Results And Discussion}

Monte-Carlo simulations (MC) are used for verification of the presented numerical analysis. Unless specified otherwise, the symbol duration $T_{s}$ is set as $1 \mu \mathrm{s}, M=1000, N=64$, $n_{1}=650, n_{2}=300, v=50, \sigma_{r}^{2}=\sigma_{d}^{2}=\sigma^{2}=0 \mathrm{~dB}, P_{\text {min }}=0.5$ $\mathrm{W}, P_{\text {max }}=9 \mathrm{~W}, d_{s r}=150 \mathrm{~m}, d_{s i}=95 \mathrm{~m}, d_{i r}=95 \mathrm{~m}$, $d_{r d}=20 \mathrm{~m}, \eta_{n}=0.2$, and RSI power $\Omega_{r r}=-10 \mathrm{~dB}$. The shape parameters for the RSI channel, $m_{r r}=3$ and $m_{s i_{n}}=$

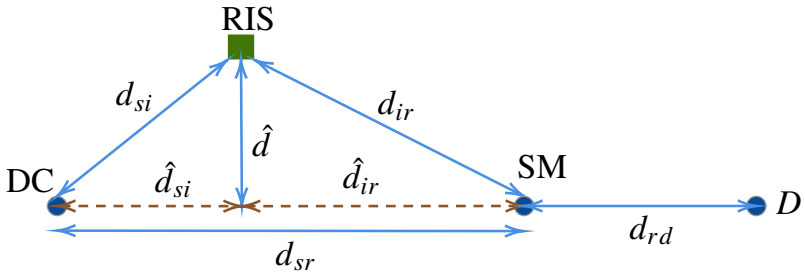

Fig. 3: Positioning of RIS

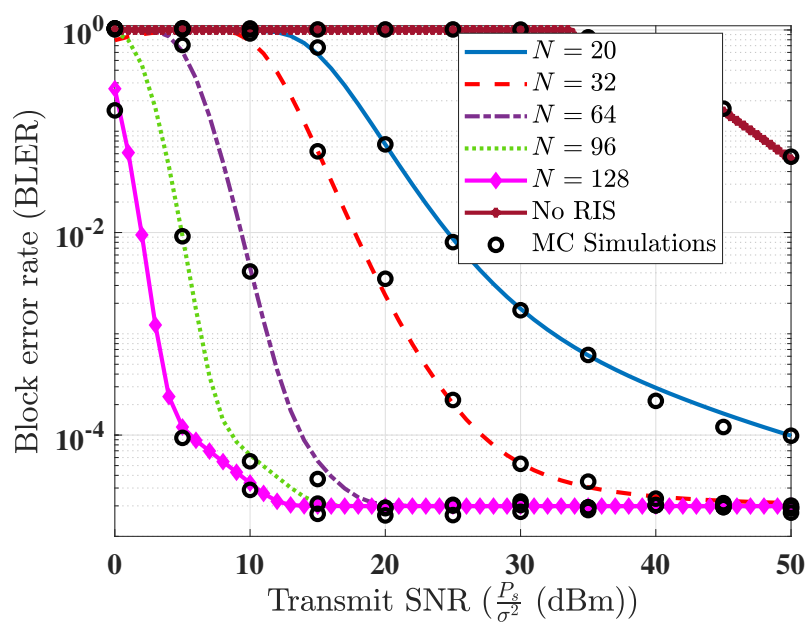

(a) BLER vs $\frac{P_{S}}{\sigma^{2}}$

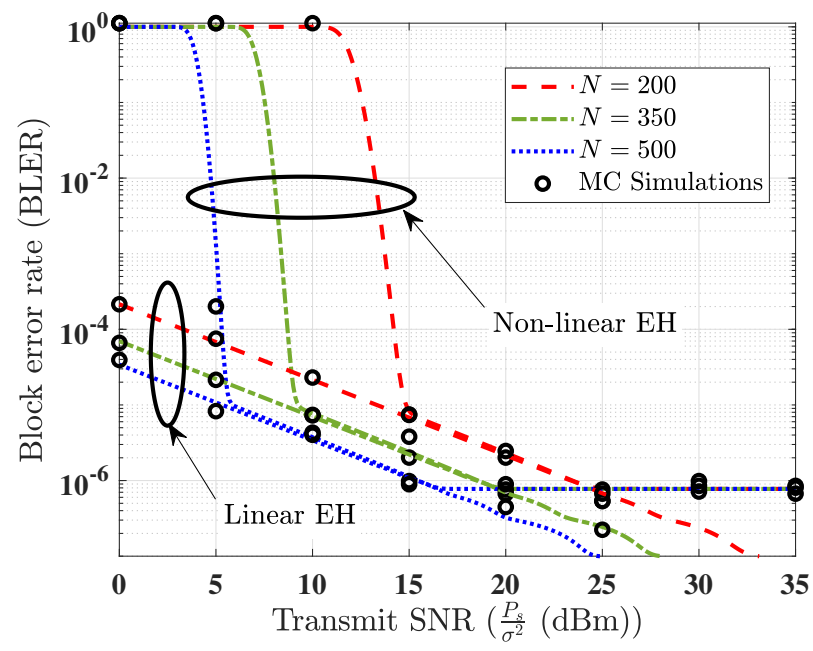

(b) BLER vs $\frac{P_{S}}{\sigma^{2}}$

Fig. 4: (a) BLER vs $\frac{P_{S}}{\sigma^{2}}$ for different number of RIS atoms compared with no RIS case. (b) BLER vs $\frac{P_{S}}{\sigma^{2}}$ for different number of RIS atoms and linear and non-linear EH.

$m_{i_{n} r}=m_{r d}=1$. The positioning of RIS follows $d_{s r}<\widehat{d}_{s i}+\widehat{d}_{i r}$ as shown in Fig. 3. We consider the path-loss model in $\mathrm{dB}$ as $\Omega=\Omega_{o}+10 v \log _{10}(d)$, where $v=3.5$ is the path-loss exponent for outdoor environment (for channels between DC to SM) and $v=2.7$ is the path-loss exponent for indoor environment (for channel from SM to $D), \Omega_{o}$ is the reference path-loss which is considered as $35 \mathrm{~dB}$ and $25 \mathrm{~dB}$ for the $\mathrm{DC}$ to $\mathrm{SM}$ and $\mathrm{SM}$ to $D$ links, respectively and $d$ is the distance. The summary of the simulation parameters is tabulated in Table II. 


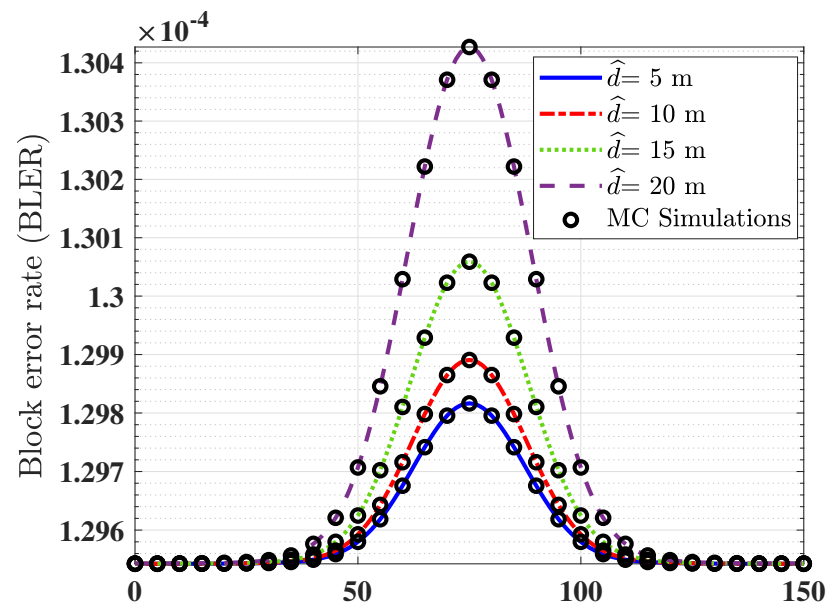

Distance between DC and RIS $\left(\widehat{d}_{s r}(\mathrm{~m})\right)$

Fig. 5: BLER vs $\widehat{d}_{s i}$ for different value of $\widehat{d}$.

\section{A. Impact of the number of RIS atoms $(N)$}

We begin with analyzing the impact of the number of RIS atoms $(N)$ on the system's performance as shown in Fig. 4a. It can be observed that when the value of $N$ increases, the performance of the system improves and it requires less $\frac{P_{s}}{\sigma^{2}}$. Moreover, the BLER for given $N$ attains approximately a common saturation. For example: when $\frac{P_{s}}{\sigma^{2}} \geq 12 \mathrm{dBm}$ and $N=128$, the BLER starts saturating. The saturation is obtained at lower values of $\frac{P_{s}}{\sigma^{2}}$, as $N$ increases which indicates that same BLER value can be obtained at lower transmit SNR if the number of RIS atoms is sufficiently increased. We further compare the performance with the direct transmission case, where no RIS is placed between the DC and SM. It can be concluded that inclusion of RIS between DC and SM significantly improves the BLER from 0.06 to a value less than $10^{-4}$, allowing transmission of control signals from a longer distance to the controlling SM. The Fig. $4 \mathrm{~b}$ shows a comparison between the non-linear and linear EH for different RIS atom. It can be observed that the linear EH leads to better reliability as compared to that for the linear $\mathrm{EH}$, but non-linear $\mathrm{EH}$ is more practical with respect to the electronic circuitry of the harvester. The BLER plots for both approximately overlap for a particular region, which corresponds to the linear region where the input power of the harvester is between sensitivity and saturation of the harvester. In order to achieve same performance for non-linear EH compared to linear EH, the system has to be operated in linear region of harvester.

\section{B. Impact of the RIS position}

Another factor that affects the system's performance is the positioning of RIS. In order to analyze its effect, the BLER performance with respect to the position of RIS $\left(\widehat{d}_{s i}\right)$ from DC is observed. We set $d_{s i}=\sqrt{\widehat{d}_{s i}^{2}+\widehat{d}^{2}}$, where $\widehat{d}$ is the perpendicular displacement of the RIS from the shortest path as illustrated in Fig. 3. Fig. 5 shows that irrespective of the values of $\widehat{d}$, the optimum performance of the system is observed if the RIS is positioned either near to DC or SM.

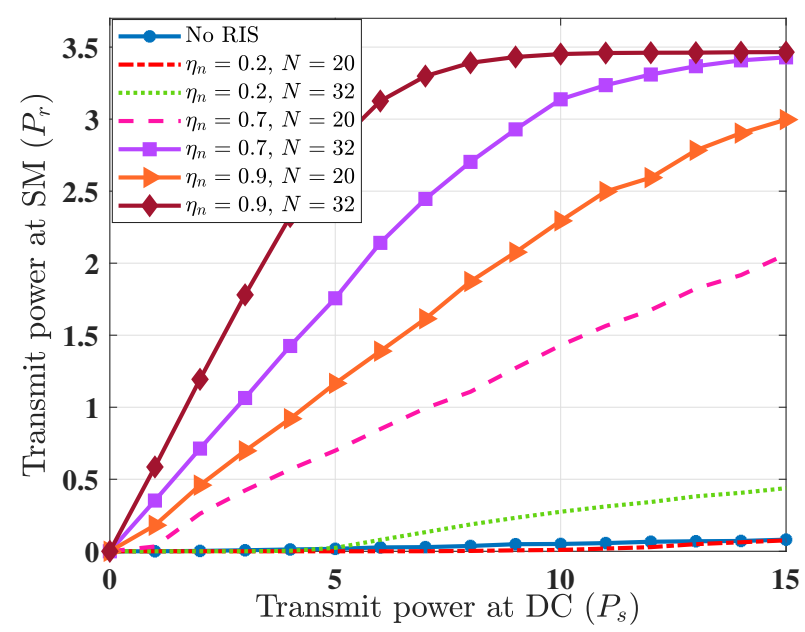

Fig. 6: Effect of RIS on energy harvesting.

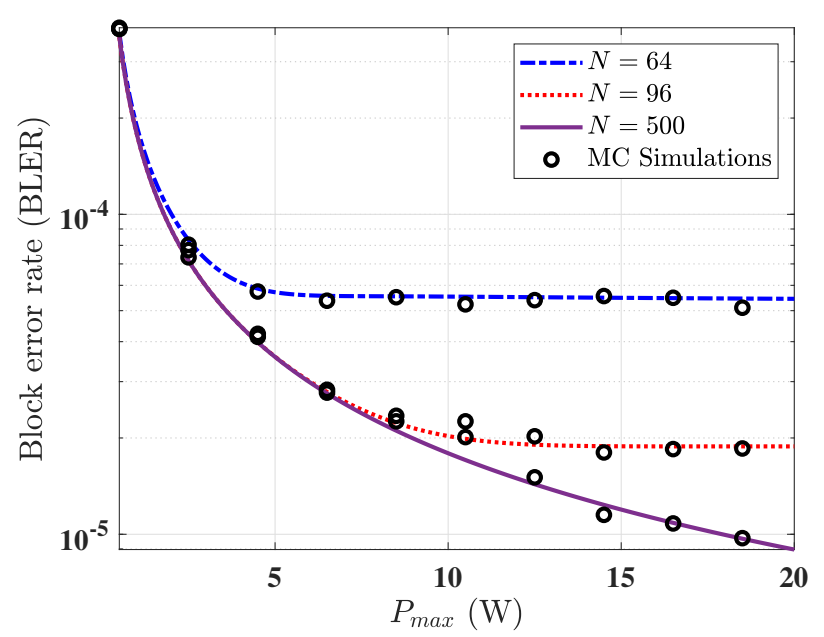

Fig. 7: BLER as a function of $P_{\max }$ for different values of $N$.

The symmetry in the curve is a result of the fact that as the distance of RIS increases from DC, it increases from SM with same factor, leading to the effective path-loss of the DC to SM channel same for $\widehat{d}_{s i}=d_{o}$ and $\widehat{d}_{i r}=d_{o}$, where $d_{o}$ is some distance between 0 to $d_{s r}$.

\section{Impact of the RIS on EH}

Further, we analyze the impact of the RIS on EH. Fig. 6 shows the transmit power of SM $\left(P_{r}\right)$ versus the transmit power of the DC $\left(P_{s}\right)$. It can be observed that for higher values of $N$ and $\eta_{n}$, the amount of harvested energy is higher as compared to the direct transmission between DC and SM and low $N$ and $\eta_{n}$ case. It can be concluded that inclusion of RIS improves the EH amount significantly. Furthermore, it can be observed that, the value of $P_{r}$ saturates to a common value due to non-linear EH for high value of $\eta_{n}$ and $N$.

\section{Impact of the saturation $\left(P_{\max }\right)$}

The variation of the BLER with respect to $P_{\max }$ is as shown in Fig. 7. It was observed that the BLER decreases with increase in $P_{\max }$ as more power is available for $\mathrm{EH}$ and hence for transmission. 


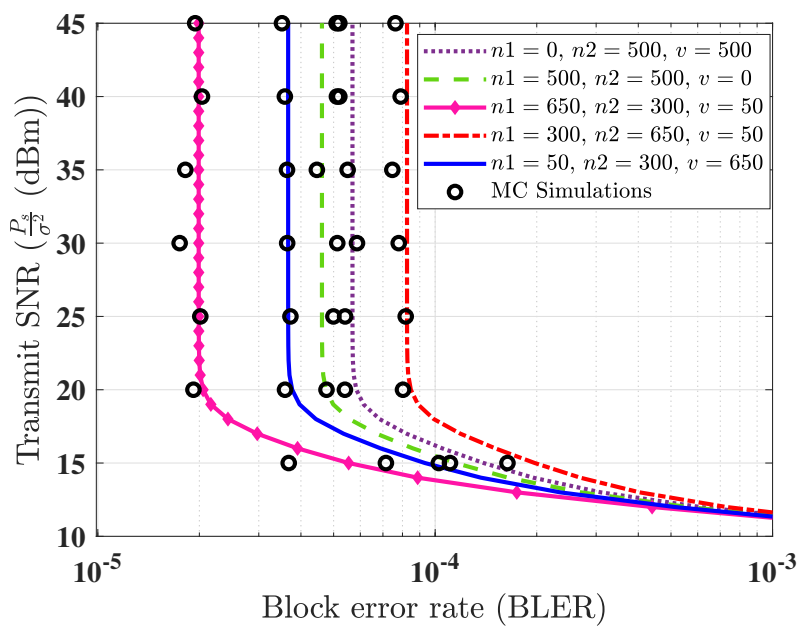

Fig. 8: $\frac{P_{S}}{\sigma^{2}}$ vs BLER for different value of $n_{1}, n_{2}$ and $v$.

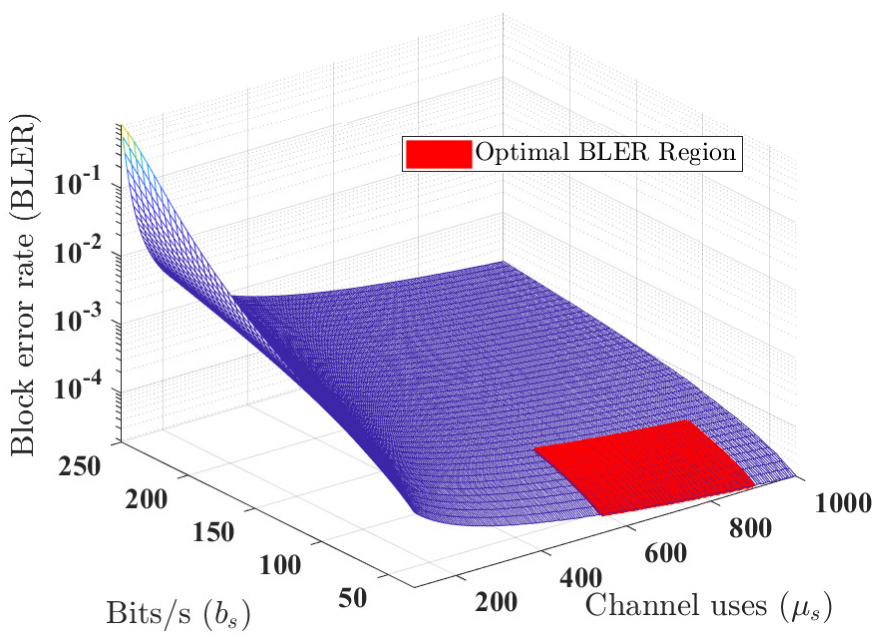

Fig. 9: Effect of the number of bits $b_{s}$ and channel uses $\mu_{s}$ on the BLER.

\section{E. Effect of EH on the BLER}

The effect of EH on the BLER is examined in Fig. 8 by varying the values of $n_{1}, n_{2}$ and $v$. Initially, we keep the TS duration constant at $v=50$ and vary $n_{1}$ and $n_{2}$. The performance improves for the case where $n_{1}>n_{2}$ due to higher time availability for ID. Similarly, for constant value of transmit duration $\left(n_{2}=300\right)$, we can observe that the improved performance is obtained for $n_{1}>v$. Further, we analyze the case of PS EH and TS EH. PS EH is achieved when the TS duration $v=0$, whereas TS EH is achieved when the PS factor $n_{1}=0$. It can be observed from Fig. 8 that the PS EH $\left(v=0, n_{1}=500, n_{2}=500\right)$ leads to slightly better performance as compared to TS EH $\left(v=500, n_{1}=0, n_{2}=500\right)$.

\section{F. Trade-off between the number of bits $b_{s}$ and channel uses $\mu_{s}$}

The trade-off between the bits/channel $\left(b_{s}\right)$ and the channel uses $\left(\mu_{s}\right)$ is observed in Fig. 9. It can be observed that the BLER performance improves for low values of $b_{s}$ and high values of $\mu_{s}$. The region for obtaining the optimal BLER lies

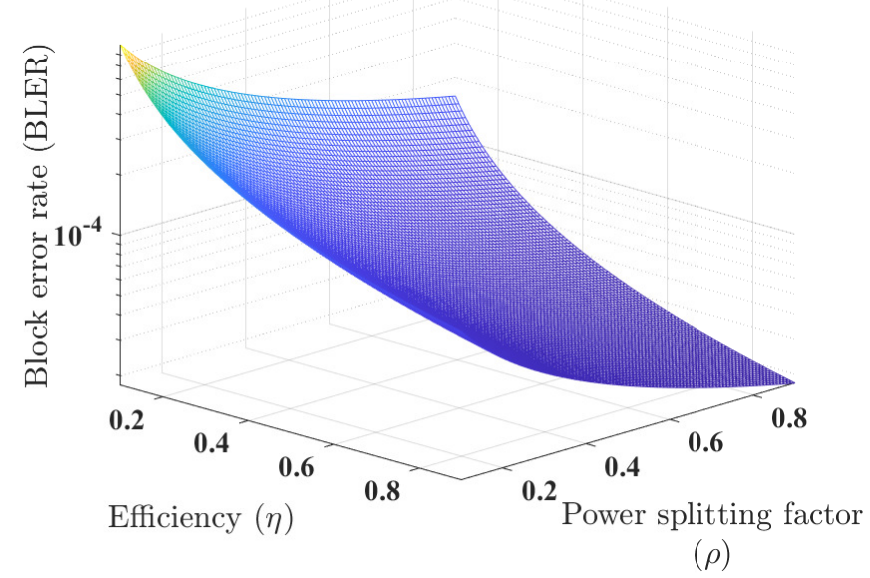

Fig. 10: Effect of the efficiency $\eta$ and PS factor $\rho$.

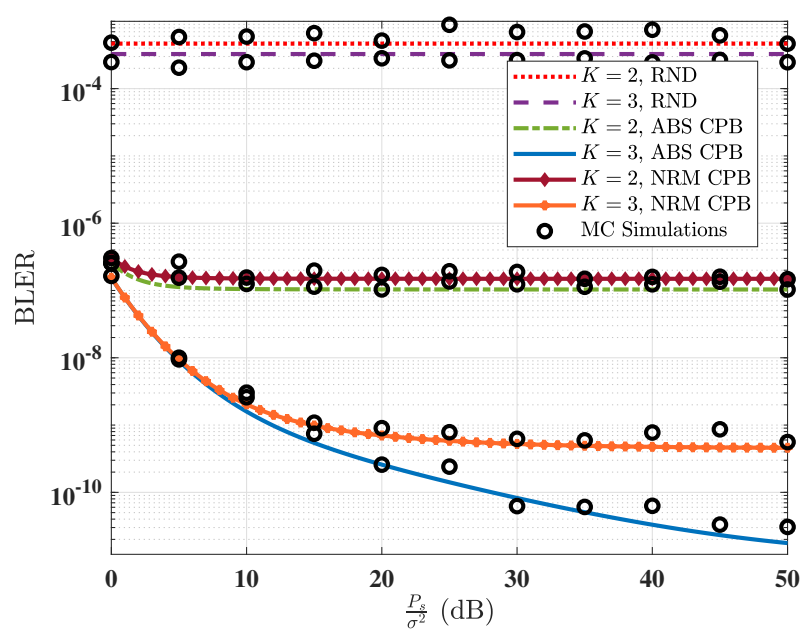

Fig. 11: BLER for different scheduling schemes and number of users.

$b_{s} \in[50,100]$ and $\mu_{s} \in[600,900]$. In Fig. 10, the trade-off between the power efficiency factor $(\eta)$ and PS ratio $(\rho)$ is illustrated. We can observe that the optimal performance is obtained at high values of both $\eta$ and $\rho$.

\section{G. Performance Comparison of Scheduling Schemes}

In order to compare different scheduling schemes, we plot the BLER vs $\frac{P_{s}}{\sigma^{2}}$ for different values of number of users $K$ and scheduling schemes as shown in Fig. 11. As can be seen that the BLER performance for all the scheduling schemes improves when $K$ increases. It can be observed that the RND scheduling scheme performs worst, followed by NRM CPB and ABS CPB. Further, the fairness of the scheduling schemes is examined in Fig. 12. It can be observed that the ABS CPB results in a lower average fairness as compared to the NRM $\mathrm{CPB}$ and the RND scheduling schemes.

\section{COnClusions And Future Works}

In this paper, we analyzed the reliability performance of an RIS-assisted and non-linear EH based wireless communication 


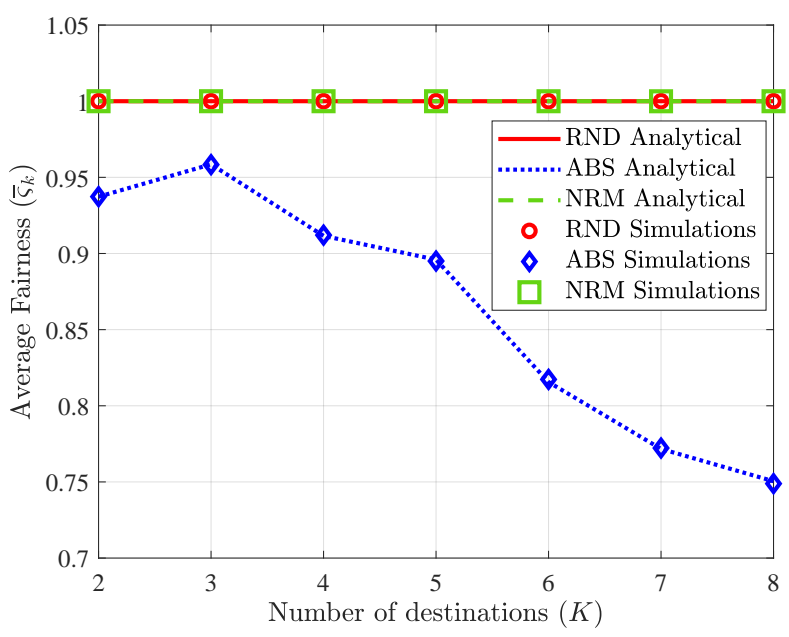

Fig. 12: Average fairness versus the number of destinations.

systems for industrial applications with URLLC constraints. We derived the end-to-end approximate closed-form expressions for the BLER and the equivalent CDF for the RND, ABS-CPB and NRM-CBP scheduling schemes. Further, we analyzed the effect of various parameters, such as number of RIS meta-atoms, blocklength, packet size and position of RIS on the system performance. Furthermore, the effect of RIS atoms on the EH was also demonstrated and noticed that the hybrid PTS EH does not guarantee the optimal performance, but leads to maximum EH. Moreover, it was observed that the BLER performance is better when the PS duration is higher as compared to the time splitting and transmission interval. The trade-off between the bits per channel and channel uses was analyzed and the optimal BLER region was determined. Moreover, we observed that for higher values of efficiency factor and PS ratio, the system becomes more reliable. The fairness of the scheduling schemes was analyzed, and it was observed that the NRM CPB and RND scheduling schemes are comparatively more fair as compared to the ABS CPB scheduling schemes. Moving towards more practical situations, we intend to consider imperfect CSI estimation for such systems in the future work.

\section{REFERENCES}

[1] "Ericsson mobility visualization: Mobility data app 2020," 2020, [Accessed on Oct. 21, 2020],. [Online]. Available: https://www. ericsson.com/en/mobility-report/mobility-visualizer

[2] "Ericsson Mobility Report, June 2020," 2020, [Accessed on Oct. 21, 2020],. [Online]. Available: https: //www.ericsson.com/49da93/assets/local/mobility-report/documents/ 2020/june2020-ericsson-mobility-report.pdf

[3] "This is 5G, Ericsson," 2019, [Accessed on Oct. 21, 2020],. [Online]. Available: https://www.ericsson.com/49df43/assets/ local/newsroom/media-kits/5g/doc/ericsson_this-is-5g_pdf_2019.pdf

[4] Q. Wu and R. Zhang, "Intelligent reflecting surface enhanced wireless network via joint active and passive beamforming," IEEE Trans. Wireless Commun., vol. 18, no. 11, pp. 5394-5409, Nov 2019.

[5] S. Loyka and G. Tsoulos, "Estimating MIMO system performance using the correlation matrix approach," IEEE Commun. Lett., vol. 6, no. 1, pp. 19-21, Jan. 2002.

[6] X. Yuan, Y.-J. A. Zhang, Y. Shi, W. Yan, and H. Liu, "Reconfigurableintelligent-surface empowered wireless communications: Challenges and opportunities," pp. 136-143, Apr. 2021.
[7] Q. Wu and R. Zhang, "Towards smart and reconfigurable environment: Intelligent reflecting surface aided wireless network," IEEE Commun. Mag., vol. 58, no. 1, pp. 106-112, Jan. 2020.

[8] Y. Hu, Y. Zhu, M. C. Gursoy, and A. Schmeink, "SWIPT-enabled relaying in IoT networks operating with finite blocklength codes," IEEE J. Sel. Areas Commun., vol. 37, no. 1, pp. 74-88, Jan 2019.

[9] P. Raut, P. K. Sharma, T. A. Tsiftsis, and Y. Zou, "Power-time splittingbased non-linear energy harvesting in FD short-packet communications," IEEE Trans. Veh. Technol., vol. 69, no. 8, pp. 9146-9151, Aug. 2020.

[10] X. Xie, J. Chen, and Y. Fu, "Outage performance and QoS optimization in full-duplex system with non-linear energy harvesting model," IEEE Access, vol. 6, pp. 44 281-44 290, Aug. 2018.

[11] C. Li, B. Xia, S. Shao, Z. Chen, and Y. Tang, "Multi-user scheduling of the full-duplex enabled two-way relay systems," IEEE Trans. Wireless Commun., vol. 16, no. 2, pp. 1094-1106, Feb. 2017.

[12] L. Yang and M. Alouini, "Performance analysis of multiuser selection diversity," IEEE Trans. Veh. Technol., vol. 55, no. 6, pp. 1848-1861, Nov. 2006.

[13] Y. Jeon, Y. Kim, M. Park, and I. Lee, "Opportunistic scheduling for multi-user two-way relay systems with physical network coding," IEEE Trans. Wireless Commun., vol. 11, no. 4, pp. 1290-1294, Apr. 2012.

[14] Y. Gu, H. Chen, Y. Li, and B. Vucetic, "Ultra-reliable short-packet communications: Half-duplex or full-duplex relaying?" IEEE Wireless Commun. Lett., vol. 7, no. 3, pp. 348-351, Jun. 2018.

[15] A. Zappone, M. Di Renzo, X. Xi, and M. Debbah, "On the optimal number of reflecting elements for reconfigurable intelligent surfaces," IEEE Wireless Commun. Lett., vol. 10, no. 3, pp. 464-468, Mar. 2021.

[16] I. Yildirim, A. Uyrus, and E. Basar, "Modeling and analysis of reconfigurable intelligent surfaces for indoor and outdoor applications in future wireless networks," IEEE Trans. Commun., vol. 69, no. 2, pp. 12901301, Feb. 2021.

[17] Z. Abdullah, G. Chen, S. Lambotharan, and J. A. Chambers, "A hybrid relay and intelligent reflecting surface network and its ergodic performance analysis," IEEE Wireless Commun. Lett., vol. 9, no. 10, pp. $1653-1657$, Oct. 2020.

[18] C. Huang, G. C. Alexandropoulos, C. Yuen, and M. Debbah, "Indoor signal focusing with deep learning designed reconfigurable intelligent surfaces," in 2019 IEEE 20th International Workshop on Signal Processing Advances in Wireless Communications (SPAWC), Jul. 2019, pp. $1-5$.

[19] J. Yuan, Y.-C. Liang, J. Joung, G. Feng, and E. G. Larsson, "Intelligent reflecting surface-assisted cognitive radio system," IEEE Trans. Commun., vol. 69, no. 1, pp. 675-687, Jan 2021.

[20] F. Challita, P. Laly, M. Yusuf, E. Tanghe, W. Joseph, M. Liénard, D. P. Gaillot, and P. Degauque, "Massive MIMO communication strategy using polarization diversity for industrial scenarios," IEEE Antennas Wireless Propag. Lett., vol. 19, no. 2, pp. 297-301, Feb. 2020.

[21] F. Shan, J. Luo, W. Wu, F. Dong, and X. Shen, "Throughput maximization for the wireless powered communication in green cities," IEEE Trans. Ind. Informat., vol. 14, no. 6, pp. 2560-2569, Jun. 2018

[22] V. K. N. Lau, S. Cai, and M. Yu, "Decentralized state-driven multiple access and information fusion of mission-critical IoT sensors for $5 \mathrm{G}$ wireless networks," IEEE J. Sel. Areas Commun., vol. 38, no. 5, pp. 869-884, May 2020.

[23] C. Huang, S. Hu, G. C. Alexandropoulos, A. Zappone, C. Yuen, R. Zhang, M. D. Renzo, and M. Debbah, "Holographic MIMO surfaces for 6G wireless networks: Opportunities, challenges, and trends," IEEE Wireless Commun., vol. 27, no. 5, pp. 118-125, Oct. 2020

[24] A. U. Makarfi, K. M. Rabie, O. Kaiwartya, O. S. Badarneh, X. Li, and R. Kharel, "Reconfigurable intelligent surface enabled IoT networks in generalized fading channels," in Proc. IEEE International Conference on Communications (ICC), Jun. 2020, pp. 1-6.

[25] P. Mursia, V. Sciancalepore, A. Garcia-Saavedra, L. Cottatellucci, X. Costa-Pérez, and D. Gesbert, "RISMA: Reconfigurable intelligent surfaces enabling beamforming for IoT massive access," IEEE J. Sel. Areas Commun., vol. 39, no. 4, pp. 1072-1085, Apr. 2021.

[26] M. Nemati, J. Ding, and J. Choi, "Short-range ambient backscatter communication using reconfigurable intelligent surfaces," in Proc. IEEE Wireless Communications and Networking Conference (WCNC), May 2020, pp. 1-6.

[27] S. Li, B. Duo, X. Yuan, Y. Liang, and M. Di Renzo, "Reconfigurable intelligent surface assisted UAV communication: Joint trajectory design and passive beamforming," IEEE Wireless Commun. Lett., vol. 9, no. 5, pp. 716-720, May 2020.

[28] Z. Peng, Z. Zhang, C. Pan, L. Li, and A. L. Swindlehurst, "Multiuser full-duplex two-way communications via intelligent reflecting surface," IEEE Trans. Signal Process., vol. 69, pp. 837-851, 2021. 
[29] N. S. Perović, L. N. Tran, M. Di Renzo, and M. F. Flanagan, "Achievable rate optimization for MIMO systems with reconfigurable intelligent surfaces," IEEE Trans. Wireless Commun., vol. 20, no. 6, pp. 38653882, Jun. 2021.

[30] A. M. Salhab and M. H. Samuh, "Accurate performance analysis of reconfigurable intelligent surfaces over Rician fading channels," IEEE Wireless Commun. Lett., vol. 10, no. 5, pp. 1051-1055, May 2021.

[31] H. Chen, G. Yang, and Y. C. Liang, "Joint active and passive beamforming for reconfigurable intelligent surface enhanced symbiotic radio system," IEEE Wireless Commun. Lett., vol. 10, no. 5, pp. 1056-1060, May 2021.

[32] X. Yu, D. Xu, and R. Schober, "Enabling secure wireless communications via intelligent reflecting surfaces," in Proc. IEEE Global Communications Conference (GLOBECOM), 2019, pp. 1-6.

[33] X. Guan, Q. Wu, and R. Zhang, "Intelligent reflecting surface assisted secrecy communication: Is artificial noise helpful or not?" IEEE Wireless Commun. Lett., vol. 9, no. 6, pp. 778-782, Jun. 2020.

[34] W. Jiang, Y. Zhang, J. Wu, W. Feng, and Y. Jin, "Intelligent reflecting surface assisted secure wireless communications with multiple- transmit and multiple-receive antennas," IEEE Access, vol. 8, pp. 86659-86673, 2020.

[35] D. Ma, G. Lan, M. Hassan, W. Hu, and S. K. Das, "Sensing, computing, and communications for energy harvesting IoTs: A survey," IEEE Commun. Surveys Tuts., vol. 22, no. 2, pp. 1222-1250, Secondquarter 2020.

[36] Y. Zheng, S. Bi, Y. J. Zhang, Z. Quan, and H. Wang, "Intelligent reflecting surface enhanced user cooperation in wireless powered communication networks," IEEE Wireless Commun. Lett., vol. 9, no. 6, pp. 901-905, Jun. 2020.

[37] B. Zheng, Q. Wu, and R. Zhang, "Intelligent reflecting surface-assisted multiple access with user pairing: NOMA or OMA?" IEEE Commun. Lett., vol. 24, no. 4, pp. 753-757, Apr. 2020.

[38] Z. Ding and H. Vincent Poor, "A simple design of IRS-NOMA transmission," IEEE Commun. Lett., vol. 24, no. 5, pp. 1119-1123, May 2020.

[39] H. Wang, C. Liu, Z. Shi, Y. Fu, and R. Song, "On power minimization for IRS-aided downlink NOMA systems," IEEE Wireless Commun. Lett., vol. 9, no. 11, pp. 1808-1811, Nov. 2020.

[40] S. Hu, F. Rusek, and O. Edfors, "Beyond massive MIMO: The potential of positioning with large intelligent surfaces," IEEE Trans. Signal Process., vol. 66, no. 7, pp. 1761-1774, Apr. 2018.

[41] G. Pan, J. Ye, J. An, and M.-S. Alouini, "When full-duplex transmission meets intelligent reflecting surface: Opportunities and challenges," 2020.

[42] D. C. Melgarejo, C. Kalalas, A. S. de Sena, P. H. J. Nardelli, and G. Fraidenraich, "Reconfigurable intelligent surface-aided grant-free access for uplink URLLC," in Proc. 2nd 6G Wireless Summit (6G SUMMIT), Mar. 2020, pp. 1-5.

[43] A. Ranjha and G. Kaddoum, "URLLC facilitated by mobile UAV relay and RIS: A joint design of passive beamforming, blocklength and UAV positioning," IEEE Internet Things J., vol. 8, no. 6, pp. 4618-4627, Mar. 2021.

[44] C. Huang, A. Zappone, G. C. Alexandropoulos, M. Debbah, and C. Yuen, "Reconfigurable intelligent surfaces for energy efficiency in wireless communication," IEEE Trans. Wireless Commun., vol. 18, no. 8, pp. 4157-4170, Aug. 2019.

[45] C. Huang, R. Mo, and C. Yuen, "Reconfigurable intelligent surface assisted multiuser MISO systems exploiting deep reinforcement learning," IEEE J. Sel. Areas Commun., vol. 38, no. 8, pp. 1839-1850, Aug. 2020.

[46] K. Singh, K. Wang, S. Biswas, Z. Ding, F. A. Khan, and T. Ratnarajah, "Resource optimization in full duplex non-orthogonal multiple access systems," IEEE Trans. Wireless Commun., vol. 18, no. 9, pp. 4312 4325, Sep. 2019.

[47] K. Singh, M. Ku, J. Lin, and T. Ratnarajah, "Toward optimal power control and transfer for energy harvesting amplify-and-forward relay networks," IEEE Trans. Wireless Commun., vol. 17, no. 8, pp. 49714986, Aug. 2018.

[48] Y. Cheng, K. H. Li, Y. Liu, and K. C. Teh, "Outage performance of downlink IRS-assisted NOMA systems," in GLOBECOM 2020 - 2020 IEEE Global Communications Conference, Dec 2020, pp. 1-6.

[49] B. Tahir, S. Schwarz, and M. Rupp, "Analysis of uplink IRS-assisted NOMA under Nakagami-m fading via moments matching," IEEE Wireless Commun. Lett., vol. 10, no. 3, pp. 624-628, Mar. 2021.

[50] D. Kudathanthirige, D. Gunasinghe, and G. Amarasuriya, "Performance analysis of intelligent reflective surfaces for wireless communication," in Proc. IEEE International Conference on Communications (ICC), 2020, pp. 1-6.
[51] L. Wei, C. Huang, G. C. Alexandropoulos, C. Yuen, Z. Zhang, and M. Debbah, "Channel estimation for RIS-empowered multi-user MISO wireless communications," IEEE Trans. Commun., vol. 69, no. 6, pp. 4144-4157, Jun. 2021.

[52] X. Wang, Z. Fei, J. Guo, Z. Zheng, and B. Li, "RIS-assisted spectrum sharing between MIMO radar and MU-MISO communication systems," IEEE Wireless Commun. Lett., vol. 10, no. 3, pp. 594-598, Mar. 2021.

[53] Z. Wang, L. Liu, and S. Cui, "Channel estimation for intelligent reflecting surface assisted multiuser communications: Framework, algorithms, and analysis," IEEE Trans. Wireless Commun., vol. 19, no. 10, pp. 66076620, Oct. 2020.

[54] C. Hu, L. Dai, S. Han, and X. Wang, "Two-timescale channel estimation for reconfigurable intelligent surface aided wireless communications," IEEE Trans. Commun., pp. 1-1, 2021.

[55] M. Abramowitz, Handbook of Mathematical Functions: With Formulas, Graphs, and Mathematical Tables. USA: Dover Publications, Inc., 1974.

[56] T. A. Khan, R. W. Heath, and P. Popovski, "Wirelessly powered communication networks with short packets," IEEE Trans. Commun., vol. 65 , no. 12, pp. 5529-5543, Dec. 2017. 